\title{
A produção científica sobre acesso no âmbito do Sistema Único de Saúde do Brasil: avanços, limites e desafios
}

\section{The scientific literature on access within the Brazilian National Health System in Brazil: advances, limitations, and challenges}

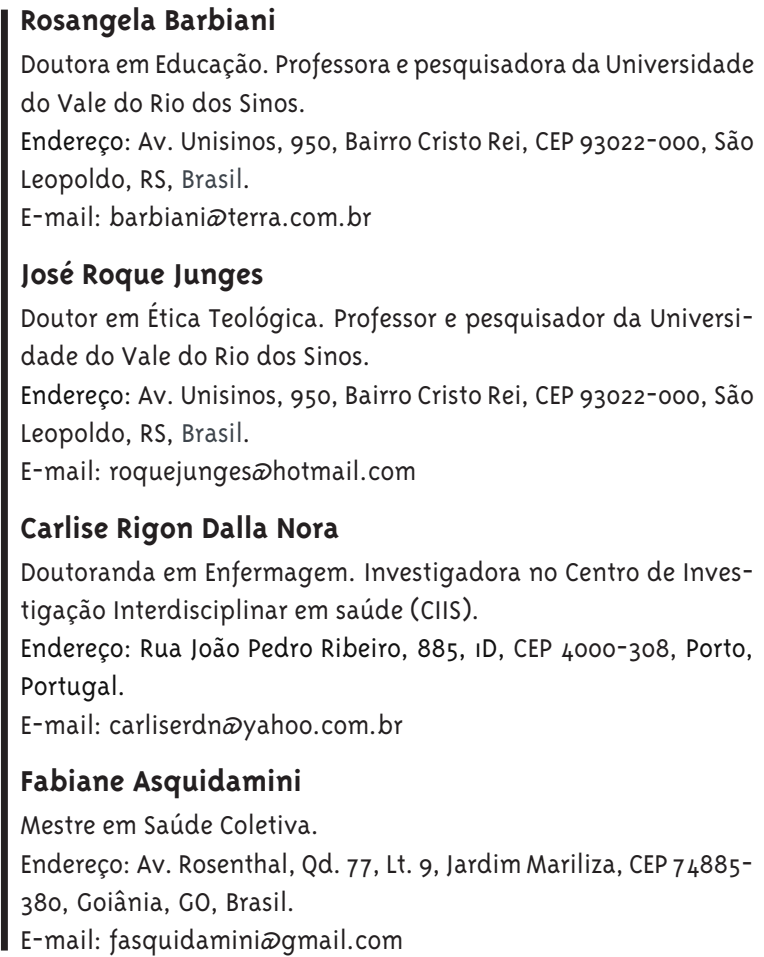

Doutoranda em Enfermagem. Investigadora no Centro de Investigação Interdisciplinar em saúde (CIIS).

Endereço: Rua João Pedro Ribeiro, 885, ID, CEP 4000-308, Porto, Portugal.

E-mail: carliserdnळyahoo.com.br

\section{Fabiane Asquidamini}

Mestre em Saúde Coletiva.

Endereço: Av. Rosenthal, Qd. 77, Lt. 9, Jardim Mariliza, CEP 74885 380, Goiânia, G0, Brasil.

E-mail: fasquidaminiळgmail.com

\section{Resumo}

A importância do acesso aos serviços de saúde para a superação dos impasses no âmbito da gestão do Sistema Único de Saúde (SUS) motivou a sistematização dos seus avanços, limites e desafios por meio de uma revisão da produção científica. Este estudo apresenta os principais resultados das pesquisas sobre acesso, tendo como universo de análise 5 revistas científicas brasileiras de Saúde Coletiva, indexadas internacionalmente e disponibilizadas na Scientific Electronic Library Online (SciELO). Contemplaram os critérios de inclusão 25 artigos envolvendo pesquisas qualitativas e relatos de experiências, escritos em português, espanhol e inglês, publicados de 2002 a 2011. Na análise do campo objetal, os resultados evidenciam a noção de acesso relacionada às seguintes categorias: processos de trabalho; organização da rede de atendimento; sistema e modelo de atenção. Centrados na avaliação das condições de acesso, os estudos direcionam as críticas aos aspectos de esgotamento do sistema, sendo a organização da rede de atendimento o principal alvo das discussões, à luz das representações sociais dos sujeitos envolvidos, em especial dos usuários dos serviços. Limites, avanços e desafios são identificados com ênfase nos níveis da micro e mesogestão, isto é, no âmbito dos processos de trabalho e na organização da rede, ficando em segundo plano a análise da lógica do sistema, definidora do modelo de atenção, que organiza o acesso aos serviços, bem como as respostas às necessidades advindas dos determinantes sociais da saúde, das transições demográficas e epidemiológicas e do papel do controle social na efetivação do direito à saúde.

Palavras-chave: Acesso aos Serviços de Saúde; Sistemas de Saúde; Atenção à Saúde. 


\section{Abstract}

The importance of access to health services in order to overcome obstacles within the management of the Brazilian National Health System gave rise to the systematization of its advances, limitations, and challenges by means of scientific literature review. This study presents the main results of researches on access, having as its universe of analysis 5 Brazilian scientific journals on Collective Health, internationally indexed and available in the Scientific Electronic Library Online (SciELO). The inclusion criteria were met by 25 articles involving qualitative researches and experience reports, written in Portuguese, Spanish, and English, published from 2002 to 2011. In the object field analysis, results show the notion of access related to the following categories: work processes; service network organization; health care system and model. Focused on assessing access conditions, the studies aim their criticism at aspects of system failure, and the service network organization is the main target of discussions, in the light of social representations of the subjects involved, particularly service users. Limitations, advances, and challenges are identified with an emphasis on the micro and meso management levels, i.e. within work processes and network organization, and the analysis of system rationale remains in the background, in spite of defining the health care model, which organizes access to services, as well as the answers to needs deriving from the social determinants of health, demographic and epidemiological transitions, and the role played by social control in the realization of the right to health. Keywords: Health Services Accessibility; Health Systems; Health Care (Public Health).

\section{Introdução}

O Sistema Único de Saúde é a organização pública de gestão e resposta à multifacetada manifestação das necessidades de saúde dos brasileiros. O sistema é a configuração estruturadora do direito constitucional da saúde como um direito de todos e um dever do Estado. A concretização desse direito está pautada pelos princípios da universalidade do acesso, da integralidade das práticas e dos processos e da equidade na distribuição dos diferentes recursos, segundo as especificidades e diferenças dos indivíduos, dos grupos e das regiões em suas necessidades. A pedra de toque da efetivação do direito à saúde e da concretização dos princípios do SUS é o acesso aos diferentes serviços e recursos, este entendido como um conceito ampliado de atenção resolutiva às necessidades de saúde de uma determinada população e território. Essa concepção extrapola o âmbito do debate puramente pragmático do acesso, direcionando-o para uma discussão teórica à luz das bases conceituais da saúde coletiva.

Nesse sentido, o acesso é uma construção social, à medida que é determinado pelo contexto sociocultural que configura as necessidades em saúde, em suas dimensões percebidas, demandadas e ofertadas (Pinheiro e Mattos, 2005). Enquanto construção social abrange e é constituído por uma multiplicidade de dimensões, apontadas por Giovanella e Fleury (1995): política, econômica, técnica e simbólica. 0 adensamento reflexivo sobre essas diferentes dimensões é proposto pelas autoras por meio de quatro modelos teóricos de explicação da categoria de acesso: economicista, sanitarista pacificador, político sanitarista e o das representações sociais. A proposta das autoras situa o acesso num quadro teórico mais amplo que aponta para a necessidade de compreendê-lo a partir do modelo de assistência que configura o sistema.

0 modelo assistencial, também definido como modelo de atenção à saúde, confere a "razão de ser”, a racionalidade, a lógica que orienta a ação, preconizando modos tecnológicos de intervenção em saúde resultantes da equalização entre necessidades, determinantes, demanda e oferta de serviços (Paim, 1999). 
Travassos e Martins (2004), em uma revisão sistemática que sintetiza a produção de vários autores internacionais, constatam que o conceito de acesso é impreciso, quando não distingue entre a oferta e o uso dos serviços. Nesse caso, sugerem a utilização da categoria acessibilidade por englobar a efetividade do acesso a partir da concepção de resolubilidade.

Hortale e colaboradores (2000) relacionam o acesso à descentralização dos serviços a partir de uma visão sistêmica. Para compreender o sistema de saúde privilegiam o modelo explicativo de Parsons, tendo como referências as instâncias central e local dos serviços, as características da população/ usuários e levando em consideração os diferentes subsistemas: o de decisão, o de operação/produção dos serviços e o de informação/retroalimentação. Contudo, essa visão funcionalista, embora seja sistêmica, não permite ver os fluxos de um sistema em rede. $\mathrm{O}$ acesso depende em grande parte da conectividade da rede.

Por isso é necessário introduzir a contribuição de Mendes (2011) sobre as redes de atenção como um caminho de explicação para os dilemas e polêmicas instituídos em torno do acesso. 0 mesmo autor apresenta uma avaliação da ineficiência do sistema de saúde para funcionar em rede. Parte da constatação de que o sistema está organizado para responder a situações de eventos agudos ou a momentos agudos de condições crônicas, quando a transição epidemiológica aponta para uma situação sanitária caracterizada predominantemente por condições crônicas, exigindo outro tipo de modelo para o atendimento e a organização dos serviços. Essa constatação sobre a atual situação epidemiológica da saúde da população e o consequente desajuste do sistema para responder às reais necessidades dos usuários tem as suas repercussões sobre a forma de conceber e organizar o acesso.

Tendo presente a multiplicidade de formas e abordagens da categoria acesso, este artigo objetiva contribuir com o debate da análise da produção científica das pesquisas qualitativas sobre o tema, tendo como chave de interpretação a explicitação de seus principais avanços, limites e desafios.

\section{Metodologia}

Foi realizada revisão da produção científica de periódicos sobre o acesso aos serviços de saúde no Brasil, por meio da busca retrospectiva de artigos publicados nos últimos dez anos, utilizando como fontes as seguintes revistas: Revista de Saúde Pública, Cadernos de Saúde Pública Ciência \& Saúde Coletiva, Interface - Comunicação, Saúde, Educação e Physis: Revista de Saúde Coletiva. Esses periódicos constituem importante fonte de informação da área científica em saúde coletiva no Brasil, encontrando-se listados em bases de indexação bibliográfica internacionais, além de disponibilizarem os seus artigos on-line, por intermédio do Projeto Scientific Electronic Library Online (SciELO).

Foram selecionados os seguintes descritores/ $\mathrm{MeSH} /$ palavras-chave de assunto: acesso, acesso aos serviços de saúde, acessibilidade aos serviços de saúde, disponibilidade de serviços de saúde, acesso aos cuidados de saúde, atenção primária em saúde-APS, modelos de atenção em saúde, demanda, necessidade em saúde e gestão.

Os estudos selecionados atenderam aos seguintes critérios de inclusão: estudos qualitativos publicados de 2002 a 2011, caracterizados como artigos de pesquisa e relatos de experiência (descrição de práticas e processos de intervenção), versando sobre o acesso aos serviços de saúde, nos idiomas inglês, espanhol ou português. Optou-se pelos estudos qualitativos por utilizarem uma variedade de práticas interpretativas interligadas na análise dos processos e significados de fenômenos socialmente construídos (Denzin e Lincoln, 2006). Por conseguinte, na abordagem do tema proposto, as pesquisas de natureza qualitativa se mostraram mais adequadas ao considerarem os aspectos subjetivos e culturais que permeiam e co-determinam as relações entre demanda, oferta e acesso, na ótica das representações sociais. Essas relações precisam ser desveladas e compreendidas, pois delas decorrem padrões de reificação de práticas, assim como rupturas e mudanças nos processos de trabalho e na gestão do sistema. Tendo presente esse pressuposto, os estudos quantitativos foram excluídos por não contemplarem as dimensões intersubjetivas do fenômeno. 
A busca bibliográfica, segundo a estratégia estabelecida, resultou em 30 artigos. Atenderam aos critérios de inclusão e foram selecionados para compor o corpus da pesquisa 25 artigos. Os outros cinco foram excluídos por se tratarem de revisões de literatura ou por serem de abordagem quantitativa. Para avaliar a qualidade dos artigos foram utilizados os critérios de classificação Qualis CAPES, na época sendo enquadrados como $\mathrm{A} 2$, $\mathrm{B} 1$ e $\mathrm{B} 2$.

Todas as publicações selecionadas foram lidas na íntegra, sendo sistematizadas suas principais características como ano, local de pesquisa e publicação, objetivos, universo empírico, sujeitos de pesquisa, coleta e análise de dados, definições de acesso, práticas relatadas e principais resultados.

Como aporte teórico à análise dos dados, utilizou-se as contribuições de Ortún Rubio (1998) e Gonzalez Garcia (2001) na definição de três esferas de gestão da saúde, caracterizadas como níveis macro, meso e microssanitários.

Para a análise os dados foram organizados em três categorias: perfil das obras, contemplando a caracterização dos estudos, campo objetal, localizando a problemática de investigação com os níveis micro, meso e macrossanitários e resultados obtidos, analisados a partir das questões de pesquisa: avanços, limites e desafios. Um mesmo artigo pode ser classificado em mais de uma categoria de análise (campo objetal e resultados).

Na análise do campo objetal em seus níveis sanitários emergiram três perspectivas sobre as quais os estudos foram categorizados: processos de trabalho (relações entre gestores, trabalhadores e usuários, associadas às práticas de cuidado e suas conexões com o acesso, vínculo e acolhimento), correspondendo ao nível microssanitário; organização da rede de saúde (determinações estruturais e de fluxos de comunicação no âmbito da rede de atendimento e de seus níveis de atenção), correspondendo ao nível mesossanitário; modelo e sistema de saúde (racionalidade que organiza a lógica da política no âmbito da gestão pública), correspondendo ao nível macrossanitário.

Em relação à análise dos resultados, a tríade avanços, limites e desafios foi correlacionada às categorias identificadas como campo objetal dos estudos. Dessa forma, operou-se com a estratégia analítica de matriciamento das categorias, no entrecruzamento de seus achados.

\section{Resultados}

\section{Caracterização do perfil das publicações}

A última década publicou poucos estudos qualitativos tematizando o acesso ao SUS, demonstrando um gap significativo, nessa abordagem, no debate científico de um tema crucial na gestão do modelo assistencial brasileiro orientado pelos princípios da universalidade, equidade e integralidade. Dos seis periódicos pesquisados, considerando as respectivas periodicidades de editoração (no total em torno de 300 números publicados na década) foi possível selecionar 25 trabalhos, dos quais $64 \%$ nos três últimos anos (2009-2011). A distribuição quantitativa dos artigos concentrou-se na revista Cadernos de Saúde Pública (12), seguida pelos periódicos Interface, Physis e Ciência \& Saúde Coletiva (quatro artigos cada um) e pela Revista de Saúde Pública (um artigo).

Se pouco expressivos em quantidade, os estudos se destacam quanto à procedência geográfica e à diversidade de temáticas exploradas. Eles abrangem grande parte das regiões do país, zonas urbanas e rurais: Norte (Amazonas); Nordeste (Bahia, Pernambuco e Rio Grande do Norte); Centro-Oeste (Mato Grosso); Sudeste (Minas Gerais, Espírito Santo, Rio de Janeiro e São Paulo) e Sul (Paraná, Santa Catarina e Rio Grande do Sul).

Seguindo a tendência de diversificação de regiões, as temáticas compuseram um amplo leque de reflexões, inclusive na forma de abordar a categoria acesso. No conjunto dos trabalhos o termo acesso e seus descritores correlatos são enunciados diretamente como problema de pesquisa em 17 artigos (Araújo e Leitão, 2005; Assis e col., 2003; Azevedo e Costa, 2010; Barbosa e Facchini, 2009; Castro e col., 2011; Coelho e Jorge, 2009; Cunha e Vieira da Silva, 2010; Ferri e col., 2007; Nogueira e col., 2007; Novo, 2011; O'Donnell, 2007; Oliveira, 2009; Oliveira e col., 2009; Ramos e Lima, 2003; Schimith e Lima, 2004; Schwartz e col., 2010; Souza e col., 2008).

Os demais artigos (Ferreira e col., 2010; Moimaz e col., 2010; Silva e col., 2010; Sisson e col., 2011; Souza e Garnelo, 2008; Tavares e col., 2009; Uchoa e col., 2011; Viegas e col., 2010) abordam a categoria como 
aspecto complementar e/ou transversal ao objeto principal de estudo, merecendo destaque os de cunho avaliativo no âmbito da satisfação da população usuária na utilização dos serviços de saúde (Silva e col., 2010; Sisson e col., 2011; Souza e Garnelo, 2008; Uchoa e col., 2011).

As referências conceituais utilizadas para definir a categoria acesso reportam-se a autores cuja produção na década de 1990 obteve grande circulação e produção de "modelos" de conceituação e caracterização das expressões acesso e acessibilidade. Nesse grupo destacam-se Fekete (1997); Unglert (1995); Donabedian (1990); Giovanella e Fleury (1995) e Travassos e Martins (2004). Da década de 2000, Starfield (2004) é a autora mais utilizada, representante da comunidade científica que analisa o fenômeno desde a ótica do modelo assistencial e de sua responsabilização na produção de iniquidades em saúde. Nesse grupo comparecem as categorias integralidade, cuidado, direito social e desigualdade social conformando o corpus teórico utilizado para a análise dos resultados das pesquisas.

Contrastando com a abrangência geográfica e a diversidade temática apresentada, os artigos evidenciam acentuada concentração quanto ao lócus e aos sujeitos de pesquisa. 0 campo de pesquisa localizou-se na atenção básica e, mais especificamente, na Estratégia Saúde da Família, sendo referido em 16 artigos (Azevedo e Costa, 2010; Barbosa e Facchini, 2009; Castro e col., 2011; Coelho e Jorge, 2009; Cunha e Vieira da Silva, 2010; Ferri e col., 2007; Moimaz e col., 2010; Novo, 2011; Oliveira e col., 2009; Ramos e Lima, 2003; Schwartz e col., 2010; Silva e col., 2010; Souza e col., 2008; Souza e Garnelo, 2008; Tavares e col., 2009; Uchoa e col., 2011). A atenção especializada em nível secundário ou terciário foi o lócus de três pesquisas (Araújo e Leitão, 2005; Assis e col., 2003; Oliveira, 2009), sendo que uma delas incluiu a atenção básica.

Essa opção é compatível com as diretrizes do modelo de atenção à saúde do SUS. Estudos apontam que a cobertura da rede de atenção básica, especificamente da Estratégia Saúde da Família, como "porta de entrada" do sistema reduz as iniquidades em saúde, além de ampliar o acesso (Becker, 2001; Rasella e col., 2010).

Quanto aos sujeitos participantes, 13 pesquisas elegeram exclusivamente os usuários (Araújo e Leitão, 2005; Assis e col., 2003; Castro e col., 2011; Ferri e col., 2007; Moimaz e col., 2010; Novo, 2011; Oliveira, 2009; Oliveira e col., 2009; Ramos e Lima, 2003; Schwartz e col., 2010; Silva e col., 2010; Sisson e col., 2011; Uchoa e col., 2011), situando-se em segundo lugar as pesquisas que incluíram o coletivo de atores da rede de atendimento (usuários, trabalhadores, gestores e conselheiros de saúde) (Azevedo e Costa, 2010; Barbosa e Facchini, 2009; Coelho e Jorge, 2009; Cunha e Vieira da Silva, 2010; Ferreira e col., 2010; Nogueira e col., 2007; Schimith e Lima, 2004; Souza e col., 2008; Souza e Garnelo, 2008; Tavares e col., 2009; Viegas e col., 2010). Houve apenas um estudo que se utilizou de banco de dados (O’Donnell, 2007).

Os resultados das pesquisas refletem a diversidade de seus enfoques e, ao mesmo tempo, um olhar analítico relativamente homogeneizado da concepção de acesso como um elemento que pode ser compreendido, em sua complexidade, no horizonte da avaliação perceptiva e representacional dos sujeitos envolvidos. Esse é o lugar sobre o qual a maioria das pesquisas partiu e, sobretudo, permaneceu, em graus diferentes de intensidade.

\section{Categorias de análise e seus resultados: avanços, limites e desafios no acesso aos serviços de saúde}

Nas abordagens ao tema os estudos identificaram os limites e desafios do SUS, em detrimento dos avanços conquistados, sinalizando que no olhar dos sujeitos envolvidos há importantes lacunas e necessidades a serem atendidas na qualidade da atenção e no acesso aos serviços no nível da atenção básica. Nessa direção, as críticas, as sugestões e os pontos positivos foram endereçados, predominantemente, aos níveis meso e microssanitários (20 artigos). A categoria processos de trabalho, correspondente ao nível microssanitário, agrupou análises relativas às relações entre gestores, trabalhadores e usuários, associadas às práticas de cuidado e suas conexões com o acesso, vínculo e acolhimento.

No nível mesossanitário, o mais destacado na referência aos limites e avanços, aqui associado à categoria organização da rede de saúde, foram agrupados os condicionantes estruturais e de fluxos de comunicação no âmbito da rede de atendimen- 
to e de seus níveis de atenção. Além dos estudos avaliativos (quatro), nessa categoria comparecem temas complexos como os contextos interculturais e a saúde da população indígena (Novo, 2011); contextos geopolíticos, como o do acesso nas linhas de fronteiras, o caso do Mercosul (Nogueira e col., 2007); contextos de gênero (Araújo e Leitão, 2005); e contextos inclusivos, como o do acesso das pessoas com deficiência (Castro e col., 2011).

Já os estudos cuja direção analítica dos resultados concentrou-se no próprio modelo e sistema de saúde analisaram as condições de acesso em relação ao nível macrossanitário, identificando contradi- ções, desafios e "cenários" nos quais as iniquidades de acesso acontecem e se originam. Sob esse enfoque foram encontrados apenas cinco artigos.

Em relação às condições restritivas ao acesso à rede de serviços, categorizadas como limites, as pesquisas apontaram um panorama crítico expressivo. Do universo estudado, 22 trazem em seus achados referências às limitações de acesso. Em consonância à caracterização dos estudos, boa parte centrada na avaliação das condições de acesso, as críticas concentram-se nos aspectos de esgotamento do sistema, sendo a organização da rede de atendimento o principal alvo das discussões (Quadro 1).

\section{Quadro I - Principais limites apontados pelas pesquisas}

\begin{tabular}{|c|c|}
\hline Cate & Prin \\
\hline $\begin{array}{l}\text { Organização da rede de atendimento } \\
\text { Araújo e Leitão, 2005; Azevedo e Costa, 2010; } \\
\text { Castro e col., 20II; Coelho e Jorge, 2009; Cunha e } \\
\text { Vieira da Silva, 2010; Ferri e col., 2007; O'Donnell, } \\
\text { 2007; Oliveira, 2009; Oliveira e col., 2009; Ramos } \\
\text { e Lima, 2003; Schwartz e col., 2010; Sisson e col., } \\
\text { 2011; Souza e col., 2008; Souza e Garnelo, 2008; } \\
\text { Tavares, e col., 2009; Uchoa e col., 20II; Viegas e } \\
\text { col., 2010. }\end{array}$ & $\begin{array}{l}\text { Relação capacidade } \text { x oferta } \times \text { demanda gerida sob a pressão e recursos de } \\
\text { cada local: demora na entrada e nos procedimentos e encaminhamentos } \\
\text { aos demais níveis de atenção, ocasionando o agravamento da condição de } \\
\text { saúde. Fila presencial sendo substituída pela virtual; coordenação da rede } \\
\text { nos territórios: fragmentação dos processos de trabalho, descontinuidade } \\
\text { do cuidado, sendo o sistema de referência contrarreferência um nó } \\
\text { crítico; serviços focalizados e seletivos; infraestrutura: número restrito de } \\
\text { trabalhadores; ausência de ações de prevenção e promoção; barreiras ao } \\
\text { acesso das pessoas com deficiência; horários de atendimento incompatíveis } \\
\text { com as possibilidades dos usuários e ausência de acolhimento à demanda } \\
\text { espontânea. }\end{array}$ \\
\hline $\begin{array}{l}\text { Processos de trabalho } \\
\text { Araújo e Leitão, 2005; Barbosa e Facchini, 2009; } \\
\text { Coelho e Jorge, 2009; Cunha e Vieira da Silva, } \\
\text { 2010; Oliveira, 2009; Ramos e Lima, 2003; Schimith } \\
\text { e Lima, 2004; Schwartz e col., 2010; Sisson e col., } \\
\text { 2011; Tavares e col., 2009; Uchoa e col., 20II }\end{array}$ & $\begin{array}{l}\text { Práticas discriminatórias nas questões de identidade e gênero (abrangendo } \\
\text { mulheres e homens); preparo técnico e conduta ética deficientes; falta de } \\
\text { comunicação entre os pares e entre os trabalhadores e usuários; concepção } \\
\text { de acolhimento como triagem; baixa valorização do contato com o paciente; } \\
\text { acessibilidade sofre grande influência das características organizacionais das } \\
\text { unidades, que traduzem os diferentes perfis profissionais e de gestão local. }\end{array}$ \\
\hline $\begin{array}{l}\text { Modelo e sistema de saúde } \\
\text { Assis e col., 2003; Azevedo e Costa, 2010; Nogueira } \\
\text { e col., 2007; Novo, 2011; Oliveira e col., 2009; } \\
\text { Schwartz e col., 2010; Sisson e col., 2011; Souza e } \\
\text { col., 2008; Souza e Garnelo, 2008; Uchoa e col., } \\
\text { 2011; Viegas e col., } 2010\end{array}$ & $\begin{array}{l}\text { Não conseguiu superar a hegemonia do modelo biomédico; a lógica } \\
\text { territorial e a inflexível definição de porta de entrada pela APS impõem aos } \\
\text { usuários uma condição em que a cidadania é dependente do seu endereço } \\
\text { de moradia; produz iniquidades: relação inversamente proporcional entre } \\
\text { situação econômica e possibilidade de acesso, exceto na questão do acesso } \\
\text { aos remédios; baixa resolutividade; não efetiva a participação dos usuários } \\
\text { na gestão (planejamento/avaliação) dos serviços; discurso que privilegia o } \\
\text { sistema de saúde privado; diversidade de compreensão sobre o significado } \\
\text { do direito à saúde; reprodução de um modelo de atenção simplificada, não } \\
\text { articulado nem com promoção, nem com a saúde como direito social. }\end{array}$ \\
\hline
\end{tabular}


A diversidade de contextos geográficos e objetais dos estudos não se reproduziu no elenco apresentado de situações-limite que envolvem o acesso da população aos serviços de saúde. Constata-se a convergência de críticas entre as categorias de análise e os níveis sanitários, confluindo para o desafio da gestão do sistema em todos os seus âmbitos como condição para a consolidação do SUS e de seus princípios.

As manifestações quanto aos avanços e às conquistas foram abordadas em apenas sete artigos, concentrando-se nas esferas de organização da rede e processo de trabalho (Quadro 2).

Os avanços apontados corroboram os achados de outros estudos representados pela ampliação do acesso e da cobertura (oferta) de serviços e de territórios (Paim e col., 2011). Essas iniciativas exitosas dizem respeito à organização da rede de atendimento e aos processos de trabalho, menos quanto ao modelo - e mesmo quando este é contemplado, relaciona-se a avanços em ações de estruturação e financiamento por incentivo do nível federal.

O conjunto dessas apreciações, sinalizando críticas e avanços, ecoa e se reproduz sob a forma de indicações de sugestões e desafios (Quadro 3).

Observa-se a recorrente convergência dessa categoria com as anteriores, com discreta precedência dos desafios atinentes aos âmbitos da organização da rede e do modelo/sistema de saúde. Contudo, os desafios e sugestões propostos pelos artigos para a melhoria do acesso não abordam a lógica do sistema definidora do modelo que organiza o acesso. Essa tendência é constatada em outras pesquisas, inclusive nas revisões de literatura sobre avaliação das redes dos serviços de saúde, ressaltando-se as poucas referências ao desempenho de sistemas no seu conjunto (Hartz e Contandriopoulos, 2004).

\section{Quadro 2 - Natureza dos avanços e seus resultados apontados pelas pesquisas}

\begin{tabular}{|l|l|}
\hline $\begin{array}{l}\text { Categorias } \\
\text { atendimento }\end{array}$ & $\begin{array}{l}\text { Principais avanços } \\
\text { Crganização do processo de adscrição dos usuários (cadastramento ativo) e a formulação } \\
\text { Ferreira e col., 20ı0; Sisson e col., } \\
\text { 20II; Uchoa e col., 20II } \\
\text { dos usuários. A desburocratização amplia o acesso; ampliação da oferta de serviços através } \\
\text { da Estratégia de Saúde da Família (ESF) e outras específicas, como a central de marcação } \\
\text { de consultas e exames especializados; centralização do gerenciamento e mapeamento da } \\
\text { demanda fornecem respostas sistematizadas às unidades; interligação dos pontos da rede } \\
\text { reorganizou o fluxo, promoveu a comunicação e a melhoria da qualidade da atenção. }\end{array}$ \\
\hline $\begin{array}{l}\text { Processos de trabalho } \\
\text { Ramos e Lima, 2003; Sisson e col., } \\
\text { col., 20I0 }\end{array}$ & $\begin{array}{l}\text { Profissionais envolvidos na prática do acolhimento; gerenciamento participativo fortalece as } \\
\text { instâncias participativas locais; aumento da comunicação horizontal entre as equipes; usuários } \\
\text { valorizam a presteza no atendimento, a qualidade técnica, o tratamento de acordo com as } \\
\text { necessidades individuais e com as diferentes subjetividades, o acesso aos cuidados necessários } \\
\text { e a cobertura oferecida; "acolhimento, vínculo e acesso" foram temas incorporados pelos } \\
\text { profissionais no desenvolvimento do seu trabalho. }\end{array}$ \\
\hline Modelo e sistema de saúde & $\begin{array}{l}\text { Investimentos na ampliação de cobertura e acessibilidade geográfica aumentam a satisfação } \\
\text { nos aspectos relacionais do cuidado, especialmente nas zonas rurais; importância do papel } \\
\text { dos profissionais da ESF e o modo como eles ajudam a população a entender a organização e o } \\
\text { cardápio de ofertas do serviço. }\end{array}$ \\
\hline
\end{tabular}




\section{Quadro 3 - Principais desafios e sugestões apontados pelas pesquisas}

\begin{tabular}{|c|c|}
\hline Categorias & Principais desafios e sugestões \\
\hline $\begin{array}{l}\text { Organização da rede de atendimento } \\
\text { Coelho e Jorge, 2009; Cunha e Vieira da Silva, } \\
\text { 2010; Ferreira e col., 2010; Ferri e col., 2007; } \\
\text { Moimaz e col., 2010; Oliveira, 2009; Schwartz e } \\
\text { col., 2010; Sisson e col., 2011; Souza e col., 2008; } \\
\text { Souza e Garnelo, 2008; Tavares e col., 2009 }\end{array}$ & $\begin{array}{l}\text { Resgatar a credibilidade e a confiabilidade no nível da APS com acesso e } \\
\text { resolutividade; ampliar cobertura da ESF e melhorar acesso (infraestrutura } \\
\text { urbana) nas áreas mais carentes; articulação da rede visando à continuidade } \\
\text { do cuidado e o equacionamento dos fluxos de referência e contrarreferência; } \\
\text { articulação intersetorial; oferta assistencial sob o gerenciamento e o controle de } \\
\text { centro regulatório, visando ao tempo satisfatório da execução do procedimento; } \\
\text { ampliação dos horários de atendimento; trabalhar na perspectiva dos } \\
\text { determinantes sociais da saúde; dar visibilidade, "reconhecer" outras realidades } \\
\text { no território, como as dos moradores em situação de rua; aumentar número de } \\
\text { trabalhadores. }\end{array}$ \\
\hline $\begin{array}{l}\text { Processos de trabalho } \\
\text { Araújo e Leitão, 2005; Barbosa e Facchini, 2009; } \\
\text { Coelho e Jorge, 2009; Ferreira e col., 2010; } \\
\text { Moimaz e col., 2010; Oliveira, 2009; Oliveira e } \\
\text { col., 2009; Tavares e col., } 2009\end{array}$ & $\begin{array}{l}\text { Discussão do processo assistencial precisa envolver o usuário, pois ele é um } \\
\text { sujeito intencional nesse processo, além de sua condição de cidadão portador } \\
\text { de direitos; organizar as equipes de modo a se adequar e reconhecer os saberes } \\
\text { e as necessidades especificas e/ou subjetivas da comunidade e dos usuários; } \\
\text { participação de trabalhadores e usuários; introduzir procedimentos que facilitem } \\
\text { a integração como o prontuário unificado; humanização do atendimento; criar } \\
\text { estratégias para evitar a exposição dos pacientes. }\end{array}$ \\
\hline $\begin{array}{l}\text { Modelo assistencial } \\
\text { Assis e col., 2003; Coelho e Jorge, 2009; Ferreira } \\
\text { e col., 2010; Ferri e col., 2007; Moimaz e col., } \\
\text { 2010; Novo, 2011; Oliveira, 2009; Oliveira e col., } \\
\text { 2009; Schwartz e col., 2010; Tavares e col., } \\
\text { 2009; Uchoa e col., 2011; Viegas e col., 2010 }\end{array}$ & $\begin{array}{l}\text { Criar mecanismos de participação social para que o processo de politização } \\
\text { promova o rompimento com o modelo de saúde centrado na saúde individual e } \\
\text { nos interesses particulares e se desloque para um entendimento de saúde pública, } \\
\text { coletiva, universal e igualitária; superação das desigualdades em termos de } \\
\text { acesso e qualidade da atenção; ampliação e inclusão no sistema de novos direitos } \\
\text { sobre uma cidadania social globalizada, fazendo eco à globalização econômica; } \\
\text { na gestão do sistema: transformar os dados em informação; sistematização } \\
\text { da avaliação como caráter institucional e instrumental do processo decisório e } \\
\text { considerar o acesso como categoria essencial para avaliar o sistema de saúde; } \\
\text { melhorar acesso aos medicamentos. }\end{array}$ \\
\hline
\end{tabular}

\section{Discussão}

Os avanços obtidos com a implementação do Sistema Único de Saúde são reconhecidos socialmente e incomparáveis com o modelo que substituiu. Trata-se de uma proposta inovadora e, sobretudo, promotora da igualdade e da equidade em saúde que resgata o direito humano à saúde de milhões de brasileiros. Ao longo de duas décadas de existência, o SUS consolidou-se como sistema que operou uma ruptura radical com um modelo marcadamente excludente, seletivo e focalizado nas demandas e interesses do mercado privado.

Na esteira desse processo histórico, a questão que está a desafiá-lo é em que medida a reforma de saúde realizada gerou também mudanças qualitativas e crescentes em seu modelo de gestão. Nesse complexo cenário inserem-se as indagações acerca do acesso aos serviços de saúde, identificadas pela pesquisa como um dos temas nevrálgicos do sistema e de sua gestão. Portanto, a discussão das questões de pesquisa que interrogam sobre os avanços, limites e desafios do acesso nas dimensões que lhe são constituintes e a partir da produção científica brasileira está necessariamente vinculada à gestão sanitária e seus respectivos níveis (micro, meso e macro) (Ortún Rubio, 1998; Gonzalez Garcia, 2001). Na concepção dos autores, o nível da macrogestão ou política sanitária é o âmbito de gestão pública em que o Estado procura regular as exigências de equidade e eficiência (sustentabilidade) do sistema. No nível meso sanitário situa-se o esforço da gestão institucional, ou seja, de coordenação dos diferentes tipos e níveis de atenção à saúde. Agregando 
as demandas de gestão da clínica, de ordenação do cuidado e dos atendimentos, cuja centralidade é a dos processos de trabalho e seus agentes, encontra-se o nível de microgestão sanitária.

Na análise dos estudos observou-se a prevalência dos níveis meso e micro em detrimento do âmbito macrossanitário, priorizando a abordagem dos limites contextuais que influenciam o acesso aos serviços. Sendo manifestações fenomênicas, ligadas ao cotidiano de vida dos usuários e de trabalho dos profissionais e gestores, esses níveis são os mais percebidos em seus limites e desafios, já que são os sujeitos participantes da maioria das pesquisas.

A identificação de limites ao acesso ocorreu em todos os níveis de atenção, desde a porta de entrada até os serviços especializados e de alta complexidade, em geral sendo associados às relações de acolhimento e vínculo, conformando-se uma visão panorâmica dos desacertos do sistema, de seus desafios, das expectativas dos usuários, como também das contradições de ambos, isto é, do sistema e dos usuários. 0 modelo de saúde é uma realidade mais invisível ao nível das estruturas do sistema, só perceptível a uma análise mais acurada que muitas vezes nem todos os gestores, profissionais e usuários estão em condições de fazer. Mas é essa lógica do sistema que configura o modelo e determina tanto a organização da rede quanto os processos de trabalho.

Diante dessa interpretação pode-se afirmar que a década passada foi tratada pelos estudos analisados como o "tempo da escuta", por meio da imersão no território dos usuários em suas relações com os trabalhadores e serviços de saúde, na busca cotidiana pelo atendimento. Nessa direção, os estudos deram "voz" e "vez" aos sujeitos, incluindo-se nesse coletivo trabalhadores e gestores, o que de certa forma explica a concentração das análises nas esferas da micro e mesogestão. $\mathrm{O}$ aspecto positivo nesses enfoques é a identificação de questões cruciais, localizadas no âmbito dos processos de trabalho e na organização da rede, passíveis de serem equacionadas nas instâncias decisórias próximas aos territórios, trabalhadores e usuários.

Entretanto, a relativa e positiva possibilidade de superação dos problemas reveste-se de outra ordem de complexidade, quando confrontada a elementos não tão visíveis e administráveis. Na análise dos entraves referidos pelas pesquisas foi mencionada como condicionante a dimensão cultural inerente aos processos históricos formativos do imaginário, das representações e da consciência dos indivíduos e da sociedade (Azevedo e Costa, 2010; Coelho e Jorge, 2009; Schimith e Lima, 2004; Silva e col., 2010; Uchoa e col., 2011). Os resultados revelam que ainda permanece, em graus diferenciados, no imaginário e na prática de trabalhadores, gestores e usuários, a visão do modelo de saúde voltado para a atenção às doenças, centrado no viés biológico. Essa perspectiva fixa posturas e concepções, dificultando as inovações e rupturas. Reforça a demanda pelas consultas médicas e a predominância do modelo queixa-conduta, fixando a figura do médico como responsável absoluto pela saúde do usuário. Nessa linha de raciocínio os usuários tendem a valorizar a qualidade do seu atendimento pelos procedimentos complexos utilizados em seu tratamento e pela facilidade em obtê-los.

A noção do direito ao acesso à saúde fica restrita à dimensão individual e às relações entre demanda e oferta. Referem as pesquisas que, embora exista consciência individual do direito à saúde, a representação é de sua não efetivação pela dificuldade de acesso aos serviços, facilitado apenas para alguns, em detrimento de outros. Essa perspectiva complexifica os entraves à responsabilização clínica e sanitária dos territórios e de suas regiões, comprometendo a ação resolutiva em situações sempre mais predominantes de condições crônicas.

Nessa racionalidade se alinham os avanços identificados pelas pesquisas na ênfase aos mecanismos que reorganizam o atendimento e os processos de trabalho. Outros mecanismos, derivados do nível macrossanitário, consoantes às políticas públicas para a mudança dessa lógica, têm dificuldade de chegar à base. A Política Nacional de Humanização e especificamente o acolhimento como diretriz e ferramenta pretendem um cambio na lógica de gestão do modelo de atendimento. Nesse sentido, e conforme Merhy (1994) e Merhy e colaboradores (1991), os modelos assistenciais são construções sociais que dizem respeito à produção de serviços de saúde e aos projetos ético-políticos a eles associados. Eles originam lógicas de atenção que transitam entre as relações de saber/poder, entre o técnico e 
o político, construtores das diretrizes de uma dada política sanitária. A possibilidade de avanços no acesso depende, em última análise, dessa política sanitária. A efetivação da humanização do cuidado implica, portanto, na reorganização do sistema e do modelo de atenção, o que torna essa mudança ainda mais complexa.

Em outras palavras, o acesso, assim como a humanização, não são tarefas exclusivas ao ethos, isto é, ao campo do ser e do fazer dos profissionais de saúde e no redirecionamento dos processos de trabalho, no âmbito da microgestão. Muito embora esse nível seja indispensável e até mesmo o "palco" das práticas humanizadas e integrais, ele depende necessariamente de arranjos estruturais de organização e gestão do sistema. Enfrentar a fragmentação dos serviços, os vazios assistenciais, as disparidades entre oferta e demanda, a carência de profissionais exige um esforço sincrônico entre os níveis macro, meso e micro, motivo pelo qual estamos diante de um desafio ético-político da constituição de redes de atenção que integrem os diferentes níveis e garantam o acesso humanizado (Junges e Barbiani, 2012).

Por isso, é pertinente assumir a análise de Mendes (2011) sobre as redes de atenção à saúde para entender a lógica que determina a demanda. Segundo ele, o sistema está organizado sob a ditadura das condições agudas e das agudizações de condições crônicas, funcionando de uma forma fragmentada, episódica e apenas reativa, forma que determina e organiza o acesso. É imprescindível, segundo o autor, mudar radicalmente essa lógica, implantando um novo sistema de atenção que, além de responder aos eventos agudos através do pronto atendimento ambulatorial e hospitalar, faça um seguimento contínuo e proativo dos portadores de condições crônicas, sob a coordenação da equipe de atenção básica. A mudança dessa lógica é uma exigência do esgotamento do sistema vigente, que não oportuniza um acesso efetivo nem organiza uma demanda resolutiva, porque não corresponde às atuais condições de saúde da população. Por isso é necessário repensar o acesso a partir de outro paradigma, que considere a transição demográfica e epidemiológica para outra situação sanitária. Esse novo cenário precisa ser debatido à luz dos eixos estruturantes do sistema que, segundo Mendes (2011), deve ser ope- rado sob a lógica das redes, o que significa pensá-lo sob a ótica da atenção primária à saúde; dos pontos de atenção secundários e terciários, ambulatoriais e hospitalares; dos sistemas de apoio; dos sistemas logísticos; e do sistema de governança.

De acordo com essa proposição, Hartz e Contandriopoulos (2004) sugerem o repensar do sistema no âmbito de sua integração às dimensões: estrutural (modificação das fronteiras das organizações), clínica (modificações nas práticas profissionais) e da cooperação (novos formatos de negociação e de acordos entre atores e organizações).

Nessa perspectiva de macroanálise constatou-se nas obras um diálogo quase inexistente com o arcabouço conceitual, normativo e operativo que vem tomando forma na gestão em todos os níveis dos entes federativos do SUS, especialmente a partir do Pacto pela Saúde (Brasil, 2006a) e da Política Nacional de Atenção Básica (Brasil, 20o6b, 2011) que indicam novas relações de contratualização entre os entes reguladores/financiadores e os prestadores de serviço. Os investimentos e as experiências que o SUS vem fazendo para a constituição das redes integradas e regionalizadas de atenção à saúde é outro exemplo desse novo tempo que se esboça promissor, ainda que a passos tímidos (Brasil, 2008, 2010). Esses caminhos que rumam à reorientação do sistema para que sejam atingidos os princípios de universalidade, integralidade e equidade precisam ser analisados pelas pesquisas para a proposição de estratégias de gestão de seus limites e desafios.

0 aprisionamento do conceito de acesso às categorizações existentes no cotidiano das práticas de saúde dificulta sua contextualização crítica na lógica organizacional que explica os seus entraves. Nesse sentido, as pesquisas dessa década contribuíram para a captura da realidade que circunscreve o acesso em suas expressões singulares constituintes. Contudo, não se pode ficar na constatação fenomênica do fato, mas tentar chaves de análise que levantem hipóteses de explicação e apontem caminhos de solução, tendo presente que as sínteses temáticas feitas a partir de revisões sistemáticas não podem reduzir-se à pura descrição da temática, mas gerar uma análise interpretativa e crítica do tema (Dixon-Woods e col., 2006). 
Por outro lado, na contextualização dos desafios postos ao sistema, destacam-se a pertinência e recorrência de alguns temas que continuam pautados na agenda da gestão: a articulação intersetorial da rede de cuidados, garantindo a continuidade do atendimento e impedindo as iniquidades no acesso; e a participação social dos atores locais, sinalizando para dimensões indissociáveis do sistema, sua estrutura organizacional e sua dinâmica complexa de rede de atores.

As desigualdades de acesso encontram-se como um dos principais problemas a serem equacionados para que o SUS funcione efetivamente, conforme os princípios e as diretrizes estabelecidos (Travassos e col., 2006; Buss e Pellegrini Filho, 2007). Nessa direção o debate deve ancorar-se na análise crítica e articulada aos outros eixos estruturantes do sistema, como o financiamento do SUS, as necessidades advindas dos determinantes sociais da saúde, das transições demográficas e epidemiológicas e do papel do controle social não apenas na luta, mas na efetivação dos direitos à saúde.

Os desafios precisam ser enfrentados com persistência e intencionalidade política pelos gestores e pela sociedade civil, a exemplo de outros já equacionados pelos SUS, exigindo pactuações em diversos níveis de regionalização, assim como investimentos que possam dar sustentação a essa mudança.

\section{Considerações finais}

Este estudo se limitou à análise das pesquisas e experiências de cariz qualitativo sobre os limites, avanços e desafios do acesso aos serviços de saúde no Brasil. Considerando essa demarcação objetal, a análise da utilização da categoria acesso permitiu a problematização de algumas questões nos campos epistemológico e metodológico da produção científica, quando aplicada à gestão de políticas públicas. Observa-se a tendência dessas produções em qualificar e situar o acesso como objeto passível de ser conhecido por meio das percepções e representações dos sujeitos envolvidos, majoritariamente dos usuários dos serviços.

Essa é uma dimensão indispensável, mas não suficiente diante da complexidade da qual se reves- te essa questão considerando o modelo da atenção preconizado pelo SUS. A realidade multifacetada e eivada de contradições exige adensamento reflexivo sobre a lógica de suas determinações estruturais e sistêmicas, contemplando a dimensão macrossanitária que estrutura o sistema de saúde. Impõe-se, portanto, um esforço coletivo de superação das formas de fragmentação que impedem o SUS de avançar, a começar pelo acesso. Essa é uma tarefa não só dos gestores e do controle social, mas também da academia.

Nessa perspectiva, decifrar as metamorfoses do presente no curso em que elas transcorrem e na urgência que o requisitam se constitui um imperativo ético e político para todos que se ocupam e se comprometem com o avanço de um Estado democrático de direito. A Constituição Cidadã de 1988 e, particularmente a configuração do SUS, apesar de suas contradições, são uma demonstração de que essa meta civilizatória é possível.

\section{Colaboração dos autores}

Barbiani participou da concepção, delineamento, análise, interpretação dos dados, redação do artigo e aprovação final da versão a ser publicada. Junges participou da concepção, delineamento, análise, revisão crítica relevante do conteúdo intelectual e aprovação final da versão a ser publicada. Dalla Nora e Asquidmini participaram da concepção, delineamento, pesquisa nas bases de dados e revisão final da versão a ser publicada.

\section{Referências}

ARAÚJO, M. A. L.; LEITÃO, G. C. M. Acesso à consulta a portadores de doenças sexualmente transmissíveis: experiências de homens em uma unidade de saúde de Fortaleza, Ceará, Brasil. Cadernos de Saúde Pública, Rio de Janeiro, v. 21, n. 2, p. 396-403, 2005.

ASSIS, M. M. A.; VILLA, T. C. S.; NASCIMENTO, M. A. A. Acesso aos serviços de saúde: uma possibilidade a ser construída na prática. Ciência \& Saúde Coletiva, Rio de Janeiro, v. 8, n. 3, p. 815$823,2003$. 
AZEVEDO, A. L. M.; COSTA, A. M. A estreita porta de entrada do Sistema Único de Saúde (SUS): uma avaliação do acesso na Estratégia de Saúde da Família. Interface: Comunicação, Saúde, Educação, Botucatu, v. 14, n. 35, p. 797-810, 2010.

BARBOSA, R. M.; FACCHINI, R. Acesso a cuidados relativos à saúde sexual entre mulheres que fazem sexo com mulheres em São Paulo, Brasil. Cadernos de Saúde Pública, Rio de Janeiro, v. 25, p. s291-s30o, 2009. Suplemento 2.

BECKER, D. No seio da família: amamentação e promoção da saúde no Programa de Saúde da Família. 2001. Dissertação (Mestrado em Saúde Pública) - Fundação Oswaldo Cruz, Escola Nacional de Saúde Pública, 2001.

BRASIL. Ministério da Saúde. Portaria n. ${ }^{0}$ 399, de 22 de feveiro de 2006. Divulga o pacto pela saúde. Diário Oficial da União, Brasília, DF, 23 fev. 2006a. Seção 1, p. 43-51.

BRASIL. Ministério da Saúde. Portaria n. ${ }^{0} 648$, de 28 de março de 2006. Aprova a Política Nacional de Atenção Básica, estabelecendo a revisão de diretrizes e normas para a organização da Atenção Básica para o Programa Saúde da Família (PSF) e o Programa Agentes Comunitários de Saúde (PACS). Diário Oficial da União, Brasília, DF, 29 mar. 2006b. Seção 1, p. 104. Disponível em: <http:// bvsms.saude.gov.br/bvs/saudelegis/gm/2006/ prto648_28_03_20o6.html >. Acesso em: 16 abr. 2013.

BRASIL. Ministério da Saúde. A estratégia de redes regionalizadas de atenção à saúde: princípios e diretrizes para sua implementação no SUS. Brasília, DF, 2008.

BRASIL. Ministério da Saúde. Portaria n. ${ }^{\circ}$ 4.279, de 30 de dezembro de 2010. Estabelece diretrizes para a organização da Rede de Atenção à Saúde no âmbito do Sistema Único de Saúde (SUS). Diário Oficial da União, Brasília, DF, 31 dez. 2010. Seção 1, p. 88.
BRASIL. Ministério da Saúde. Portaria n. ${ }^{0} 2.488$, de 21 de outubro de 2011. Política Nacional de Atenção Básica, estabelecendo a revisão de diretrizes e normas para a organização da Atenção Básica, para a Estratégia Saúde da Família (ESF) e o Programa de Agentes Comunitários de Saúde (PACS). Diário Oficial da União, Brasília, DF, 24 out. 2011. Seção 1, p. 48.

BUSS, P. M.; PELLEGRINI FILHO, A. A saúde e seus determinantes sociais. Physis: Revista de Saúde Coletiva, Rio de Janeiro, v. 17, n. 1, p. 77-73, 2007.

CASTRO, S. S. et al. Acessibilidade aos serviços de saúde por pessoas com deficiência. Revista de Saúde Pública, São Paulo, v. 45, n. 1, p. 99-105, 2011.

COELHO, M. O.; JORGE, M. S. B. Tecnologia das relações como dispositivo do atendimento humanizado na atenção básica à saúde na perspectiva do acesso, do acolhimento e do vínculo. Ciência \& Saúde Coletiva, Rio de Janeiro, v. 14, p. 1523-1531, 2009. Suplemento.

CUNHA, A. B. O.; VIEIRA DA SILVA, L. M. Acessibilidade aos serviços de saúde em um município do Estado da Bahia, Brasil, em gestão plena do sistema. Cadernos de Saúde Pública, Rio de Janeiro, v. 26, n. 4, p. 725-737, 2010.

DENZIN, N. K.; LINCOLN, Y. S. O planejamento da pesquisa qualitativa: teorias e abordagens. Porto Alegre: Artmed, 2006.

DIXON-WOODS, M. et al. Conducting a critical interpretive synthesis of the literature on access to healthcare by vulnerable groups. BMC Medical Research Methodology, London, v. 6, n. 35, p. 1-13, 20o6. Disponível em: <http://www.biomedcentral. com/content/pdf/1471-2288-6-35.pdf >. Acesso em: 11 fev. 2013.

DONABEDIAN, A. The seven pillars of quality. Archives of Pathology Laboratory Medicine, Chicago, v. 114, n. 11, p. 1115-1118, 1990.

FEKETE, M. C. Estudo da acessibilidade na avaliação dos serviços de saúde. In: SANTANA, J. P. Desenvolvimento gerencial de unidades básicas do Sistema Único de Saúde (SUS). Brasília, DF: OPAS, 1997. p. 236-251. 
FERREIRA, J. B. B. et al. O complexo regulador da assistência à saúde na perspectiva de seus sujeitos operadores. Interface: Comunicação, Saúde, Educação, Botucatu, v. 14, n. 33, p. 345-358, 2010.

FERRI, S. M. N. et al. As tecnologias leves como geradoras de satisfação em usuários de uma unidade de saúde da família. Interface: Comunicação, Saúde, Educação, Botucatu, v. 11, n. 23, p. 515-529, 2007.

GIOVANELLA, L.; FLEURY, S. Universalidade da atenção à saúde: acesso como categoria de análise. In: EIBENSCHUTZ, C. Política de saúde: o público e o privado. Rio de Janeiro: Fiocruz, 1995. p. 177-198.

GONZALEZ GARCIA, G. Las reformas sanitarias y los modelos de gestión. Revista Panamericana Salud Publica, Washington, DC, v. 9, n. 6, p. 406412, 2001.

HARTZ, Z. M. A.; CONTANDRIOPOULOS, A. P. Integralidade da atenção e integração de serviços de saúde: desafios para avaliar a implantação de um "sistema sem muros". Cadernos de Saúde Pública, Rio de Janeiro, v. 20, p. 331-336, 2004. Suplemento 2.

HORTALE, V. A.; PEDROZA, M.; ROSA, M. L. G. Operacionalizando as categorias acesso e descentralização na análise de sistemas de saúde. Cadernos de Saúde Pública, Rio de Janeiro, v. 16, n. 1, p. 231-239, 2000.

JUNGES, J. R.; BARBIANI, R. Repensando a humanização do Sistema Único de Saúde à luz das redes de atenção à saúde. $O$ Mundo da Saúde, São Paulo, v. 36, n. 3, p. 397-406, 2012.

MENDES, E. V. As redes de atenção à saúde. Brasília, DF: OPAS, 2011.

MERHY, E. E.; CECÍLIO, L. C. O; NOGUEIRA FILHO, R. C. Por um modelo tecno-assistencial da política de saúde em defesa da vida: contribuição para as conferências de saúde. Saúde em Debate, Rio de Janeiro, n. 33, p. 83-89, dez. 1991.

MERHY, E. E. Em busca da qualidade dos serviços de saúde: os serviços de porta aberta para a saúde e o modelo tecnoassistencial em defesa da vida. In: CECILIO, L. C. D. O. Inventando a mudança em saúde. São Paulo: Hucitec, 1994. p. 117-16o.
MOIMAZ, S. A. S. et al. Satisfação e percepção do usuário do SUS sobre o serviço público de saúde. Physis: Revista de Saúde Coletiva, Rio de Janeiro, v. 20, n. 4, p. 1419-1440, 2010.

NOGUEIRA, V. M. R.; DAL PRÁ, K. R.; FERMIANO, $\mathrm{S}$. A diversidade ética e política na garantia e fruição do direito à saúde nos municípios brasileiros da linha da fronteira do Mercosul. Cadernos de Saúde Pública, Rio de Janeiro, v. 23, p. 227-236, 2007. Suplemento 2.

NOVO, M. P. Política e intermedicalidade no Alto Xingu: do modelo à prática de atenção à saúde indígena. Cadernos de Saúde Pública, Rio de Janeiro, v. 27, n. 7, p. 1362-1370, 2011.

O'DONNELL, O. Access to health care in developing countries: breaking down demand side barriers. Cadernos de Saúde Pública, Rio de Janeiro, v. 23, n. 12, p. 2820-2834, 2007.

OLIVEIRA, I. B. N. Acesso universal?: obstáculos ao acesso, continuidade do uso e gênero em um serviço especializado em HIV/AIDS em Salvador, Bahia, Brasil. Cadernos de Saúde Pública, Rio de Janeiro, v. 25, p. 259-268, 2009. Suplemento 2.

OLIVEIRA, L. H.; MATTOS, R. A.; SOUZA, A. I. S. Cidadãos peregrinos: os "usuários" do SUS e os significados de sua demanda a prontos-socorros e hospitais no contexto de um processo de reorientação do modelo assistencial. Ciência \& Saúde Coletiva, Rio de Janeiro, v. 14, n. 5, p. 19291938, 2009.

ORTÚN RUBIO, V. Gestión sanitaria y calidad. In: CONGRESO NACIONAL DE LA SOCIEDAD ESPAÑOLA DE CALIDAD ASISTENCIAL, 1998, Santander. Barcelona, 1998. p. 1-9. Disponível em: <http://www.econ.upf.edu/ ortun/publicacions/ paper1o.pdf $>$. Acesso em: 20 fev. 2013.

PAIM, J. S. A reforma sanitária e os modelos assistenciais. In: ROUQUAYROL, M. Z.; ALMEIDA FILHO, N. Epidemiologia \& saúde. 5. ed. Rio de Janeiro: Medsi, 1999. p. 473-487.

PAIM, J. et al. Saúde no Brasil: o sistema de saúde brasileiro: história, avanços e desafios. The Lancet, London, v. 377, n. 9779, p. 11-31, 2011. 
PINHEIRO, R.; MATTOS, R. A. Construção social da demanda: direito à saúde, trabalho em equipe, participação e espaços públicos. 2. ed. Rio de Janeiro: Abrasco, 2005.

RAMOS, D. D.; LIMA, M. A. D. S. Acesso e acolhimento aos usuários em uma unidade de saúde de Porto Alegre, Rio Grande do Sul, Brasil. Cadernos de Saúde Pública, Rio de Janeiro, v. 19, n. 1, p. 27-34, 2003.

RASELLA, D.; AQUINO, R.; BARRETO, M. L. Impact of the Family Health Program on the quality of vital information and reduction of child unattended deaths in Brazil: an ecological longitudinal study. BMC Public Health, London, v. 10, p. 380, June, 2010. Disponível em: <http://www. biomedcentral.com/content/pdf/1471-2458-10-38o. pdf $>$. Acesso em: 15 fev. 2013.

SCHIMITH, M. D.; LIMA, M. A. D. S. Acolhimento e vínculo em uma equipe do Programa Saúde da Família. Cadernos de Saúde Pública, Rio de Janeiro, v. 20, n. 6, p. 1487-1494, 2004.

SCHWARTZ, T. D. et al. Estratégia Saúde da Família: avaliando o acesso ao SUS a partir da percepção dos usuários da unidade de saúde de Resistência, na região de São Pedro, no município de Vitória (ES). Ciência \& Saúde Coletiva, Rio de Janeiro, v. 15, n. 4, p. 2145-2154, 2010.

SILVA, M. A. M.; FERREIRA, E. F.; SILVA, G. A. O direito à saúde: representações de usuários de uma unidade básica de saúde. Physis: Revista de Saúde Coletiva, Rio de Janeiro, v. 20, n. 4, p. 11831207, 2010.

SISSON, M. C. et al. Satisfação dos usuários na utilização de serviços públicos e privados de saúde em itinerários terapêuticos no sul do Brasil. Interface: Comunicação, Saúde, Educação, Botucatu, v. 15, n. 36, p. 123-136, 2011.
SOUZA, E. C. F. et al. Acesso e acolhimento na atenção básica: uma análise da percepção dos usuários e profissionais de saúde. Cadernos de Saúde Pública, Rio de Janeiro, v. 24, p. s1oo-s11o, 2008. Suplemento 1.

SOUZA, M. L. P.; GARNELO, L. "É muito dificultoso!": etnografia dos cuidados a pacientes com hipertensão e/ou diabetes na atenção básica, em Manaus, Amazonas, Brasil. Cadernos de Saúde Pública, Rio de Janeiro, v. 24, p. s91-s99, 2008. Suplemento 1.

STARFIELD, B. Atenção primária: equilíbrio entre necessidades de saúde, serviços e tecnologia. Brasília, DF: Unesco: Ministério da Saúde, 2004.

TAVARES, M. D. F. L.; MENDONÇA, M. H. M.; ROCHA, R. M. Práticas em saúde no contexto de reorientação da atenção primária no Estado do Rio de Janeiro, Brasil, na visão das usuárias e dos profissionais de saúde. Cadernos de Saúde Pública, Rio de Janeiro, v. 25, n. 3, p. 1054-1062, 2009.

TRAVASSOS, C.; MARTINS, M. Uma revisão sobre os conceitos de acesso e utilização de serviços de saúde. Cadernos de Saúde Pública, Rio de Janeiro, v. 20, p. s19o-s198, 2004. Suplemento 2.

TRAVASSOS, C.; OLIVEIRA, E. X. G.; VIACAVA, F. Desigualdades geográficas e sociais no acesso aos serviços de saúde no Brasil: 1998 e 2003. Ciência ¿ Saúde Coletiva, Rio de Janeiro, v. 11, n. 4, p. 975986, 2006.

UCHOA, A. C. et al. Avaliação da satisfação do usuário do Programa de Saúde da Família na zona rural de dois pequenos municípios do Rio Grande do Norte. Physis: Revista de Saúde Coletiva, Rio de Janeiro, v. 21, n. 3, p. 1061-1076, 2011.

UNGLERT, C. V . S. Territorialização em sistemas de saúde. In: MENDES, E. V. (Org.). Distrito sanitário. São Paulo: Hucitec, 1995 p. 221-235.

VIEGAS, S. M. F. et al. O cotidiano da assistência ao cidadão na rede de saúde de Belo Horizonte. Physis: Revista de Saúde Coletiva, Rio de Janeiro, v. 20, n. 3, p. 769-784, 2010.

Recebido em: 16/04/2013

Reapresentado em: 08/10/2013

Aprovado em: $12 / 11 / 2013$ 